\title{
Optimal Dispatch in Power Systems with Intermittent Power Sources
}

\author{
Lai Peng \\ Wuxi Big Bridge Academy, Wuxi, China \\ Email: penglai222@outlook.com
}

How to cite this paper: Peng, L. (2020) Optimal Dispatch in Power Systems with Intermittent Power Sources. Computational Water, Energy, and Environmental Engineering, 9, 101-107.

https://doi.org/10.4236/cweee.2020.94008

Received: September 26, 2019

Accepted: August 29, 2020

Published: September 1, 2020

Copyright $\odot 2020$ by author(s) and Scientific Research Publishing Inc. This work is licensed under the Creative Commons Attribution International License (CC BY 4.0).

http://creativecommons.org/licenses/by/4.0/

\begin{abstract}
With the increasing integration of intermittent power sources (IPSs) into the power system, the uncertainty of IPSs requires solution and current dispatch system needs improvement. This paper aims to generate the optimal dispatch plan for day-ahead scheduling and real-time dispatch using the proposed model of characteristic optimal power flow (COPF). The integral time period represented by the median load point and the heavy and light load point with simplicity and accuracy. Simulation case studies on a 30-bus system are presented, which shows that COPF is an effective model to generate the optimal dispatch plan for power systems with high penetration of IPSs.
\end{abstract}

\section{Keywords}

Intermittent Power Sources, Dispatch System, Characteristic Optimal Power Flow

\section{Introduction}

Due to the energy crisis, intermittent power sources (IPSs), such as wind and solar power sources, are considered as the primary and promising renewable energy sources. Compared with traditional power sources, IPSs are more environmentally friendly [1]. Therefore, IPSs have been widely integrated and growing into the power systems. More specifically, the first few countries in terms of wind power proportion since 2016 are respectively given by [2]: 1) Denmark: $40 \%$, 2) Uruguay: $23.5 \%, 3)$ Spain: $20 \%$.

Despite the rapid development in IPS power generations, IPS has its own drawbacks. Due to the IPS power uncertainty, the power balancing process and the system's security (e.g. voltage quality) may be jeopardized [3]. Hence, to solve the IPSs uncertainty problem, there are 3 main ways to go. First, to enhance different forecasting methods reduces the forecasting error [4]. Second is 
accommodation, which means to allocate the accommodation reserves from controllable power sources to deal with uncertainties [5]. Third is adjustment, which means to adjust the current dispatch control systems from power balancing perspective [6]. In power systems with high penetrations of IPSs, from the power balancing perspective, the dispatch control system is divided into the following 3 levels: day-ahead scheduling, real-time dispatch, and automatic generation control (AGC) [7]. Day-ahead scheduling adjusts the controllable power sources to balance day-ahead forecast value and arrange accommodation and AGC reserves [8]. Real-time dispatch corrects the "vague" day-ahead forecasting with the "clear" real time forecasting value, and balances the real time forecasting value with the accommodation reserve [9]. AGC corrects the "clear" real time forecasting value with the accurate actual value, and balances the actual value with the AGC reserve [10].

The optimal power flow (OPF) tool that was first proposed by Carpentier [11] has been widely applied to generate the optimal generation schedules under each level of dispatch control system. There are many OPF algorithms such as Newton method and the Interior-point method to deal with the inequality constraints. Traditional optimal power flow (TOPF) aims to find the optimal solution in power systems with the following general formula:

$$
\begin{array}{ll}
\min & f(x, u) \\
\text { s.t. } & g(x, u)=0 \\
& h(x, u) \leq 0
\end{array}
$$

where $f(x)$ is the objective function that represents the total cost of fuel or the power loss, $g(x)$ is the equality constraint set that represents the power flow equation, and $h(x)$ is the inequality constraint set that typically includes the node voltage constraints, generator constraints and branch capacity constraints, $u$ is controllable variable set that normally represents the active power output of generators, and $x$ is the state variable set that includes the each voltage magnitude and phase angle under the polar coordinates.

To generate the optimal generation schedules in each dispatch level, including day-ahead scheduling and real-time dispatch, TOPF calculates value at a particular single point and then applies its results to a time period. However, since power system conditions are towards temporal constraints, thus a particular single point cannot represent the whole time period. More specifically, power flow distributions are not identical at all points within a given time period, constraints satisfied at the particular single point do not guarantee the same constraints to be satisfied at any other points, especially considering the binding constraints within a time period. Therefore, our COPF model takes the median load point, heavy and light load points into consideration, improves on the basis of the TOPF model, and proposes the COPF model.

This paper aims to use characteristic optimal power flow (COPF) [12] to generate the optimal generation scheduling plans, including the day-ahead scheduling and real time dispatch, for power systems with high penetrations of IPSs. 
The remainder of the paper is as follows. Systems modelling about power systems with high penetrations of IPSs is given in Section 2. Simulation results on IEEE 30-bus system are tested and recorded in Section 3. Conclusions are finally given in Section 4.

\section{Systems Modeling}

\subsection{Net Load}

The IPS uncertainties of power generation output depends on from different environmental conditions. Since the traditional loads have uncertainties, the IPSs can be also considered as negative loads. Thus, we define net load as the combination of traditional loads and IPSs (negative loads), and is further given by:

$$
P_{n e t}=P_{l}+P_{i p s}
$$

where $P_{n e t}$ represents the power generation from net/equivalent loads; $P_{l}$ is the traditional load and $P_{i p s}$ is the negative loads from IPSs (wind/solar power).

\subsection{Nodal Power Flow Equation}

The total power generation, including both active and reactive power, needs to be balanced by the total power loss and the total loads in the power networks. Let $Q_{G}, Q_{G}$ denote the generation power of bus $i, P_{n e t}^{i}, Q_{n e t}^{i}$ denote the net power of load bus $i$, and $P_{\text {loss }}^{i}, Q_{\text {loss }}^{i}$ denote the power loss on a branch.

$$
\begin{aligned}
& \sum_{i=1}^{n} P_{G}^{i}=\sum_{i=1}^{n} P_{\text {net }}^{i}+\sum_{i=1}^{n} P_{\text {loss }}^{i} \\
& \sum_{i=1}^{n} Q_{G}^{i}=\sum_{i=1}^{n} Q_{\text {net }}^{i}+\sum_{i=1}^{n} Q_{\text {loss }}^{i}
\end{aligned}
$$

where $n$ is the number of buses in the network.

\subsection{Median Load Point}

According to COPF model, we take the median load point as the objective function of OPF, and according to the mean-value theorem, the rigorous integral OPF that is orient to the time period can be represented as the following equation which is focused on the median load point:

$$
\int_{t_{0}}^{t_{1}} f(x(t), u) \mathrm{d} t=f\left(x_{m}, u_{m}\right) \times\left(t_{1}-t_{0}\right)
$$

where $t_{0}$ and $t_{1}$ represent the beginning point and ending point of a given time period, respectively; $x_{m}$ and $u_{m}$ represent the state variables and control variables at median point, respectively.

\subsection{Heavy and Light Load Points}

In the COPF model, the two extreme loading conditions of a given period are characterized as the heavy and light load points, respectively. Thus, the two corresponding loading points are applied to check the inequality constraints. COPF guarantees that, as long as the constraints on heavy and light load points are sa- 
tisfied, the constraints on any other load points within a given time period are also satisfied. In other words, no constraints violations will occur on the given time period.

\subsection{Characteristic Optimal Power Flow Model}

Based on our previous analysis, the complete COPF that characterizes three different load points within a time period is presented as follows.

1) The objective function is calculated at the median load point as follows, which generally aims to obtain the minimum generation cost in traditional generators:

$$
\min f\left(x_{m}, u_{m}\right) \times \Delta T
$$

where $\Delta T$ represents the time length of the given time period, for example 30 mins or $1 \mathrm{~h}$.

2) The nodal equality constraints need to be satisfied at three characterized points: the median load point, the heavy load point and the light load point:

$$
\begin{aligned}
& \boldsymbol{g}\left(x_{m}, u_{m}\right)=0 \\
& \boldsymbol{g}\left(x_{\mathrm{ma}}, u_{\mathrm{ma}}\right)=0 \\
& \boldsymbol{g}\left(x_{\mathrm{mi}}, u_{\mathrm{mi}}\right)=0
\end{aligned}
$$

where $x_{\mathrm{ma}}$ and $u_{\mathrm{ma}}$ represent the state variables and control variables on the heavy load point, $x_{\mathrm{mi}}$ and $u_{\mathrm{mi}}$ represent the state variables and control variables on the light load point.

3) The inequality constraints are evaluated at the heavy and light load points as follows:

$$
\begin{aligned}
& \boldsymbol{h}\left(x_{\mathrm{ma}}, u_{\mathrm{ma}}\right) \leq 0 \\
& \boldsymbol{h}\left(x_{\mathrm{mi}}, u_{\mathrm{mi}}\right) \leq 0
\end{aligned}
$$

More specifically, the inequality constraints normally include voltage inequality constraints, the controllable power inequality constraints and the line power inequality constraints:

$$
\begin{gathered}
\left\{\begin{array}{l}
\boldsymbol{V}_{\min } \leq \boldsymbol{V}_{i}^{\mathrm{ma}} \leq \boldsymbol{V}_{\max } \\
\boldsymbol{V}_{\min } \leq \boldsymbol{V}_{i}^{\mathrm{mi}} \leq \boldsymbol{V}_{\max }
\end{array}\right. \\
\left\{\begin{array}{l}
\boldsymbol{P}_{\min }^{c} \leq \boldsymbol{P}_{i}^{\mathrm{ma}} \leq \boldsymbol{P}_{\max }^{c} \\
\boldsymbol{Q}_{\min }^{c} \leq \boldsymbol{Q}_{i}^{\mathrm{ma}} \leq \boldsymbol{Q}_{\max }^{c} \\
\boldsymbol{P}_{\min }^{c} \leq \boldsymbol{P}_{i}^{\mathrm{mi}} \leq \boldsymbol{P}_{\max }^{c} \\
\boldsymbol{Q}_{\min }^{c} \leq \boldsymbol{Q}_{i}^{\mathrm{mi}} \leq \boldsymbol{Q}_{\max }^{c}
\end{array}\right. \\
\left\{\begin{array}{l}
\boldsymbol{P}_{\operatorname{mi}}^{\text {line }} \leq \boldsymbol{P}_{\max }^{\text {line }} \\
\boldsymbol{P}_{\operatorname{ma}}^{\text {line }} \leq \boldsymbol{P}_{\max }^{\text {line }}
\end{array}\right.
\end{gathered}
$$

\section{Simulation Results}

\subsection{Simulation System}

In this section, IEEE 30-bus system with IPSs is tested to verify the proposed 
COPF. Wherein, bus 4 is added by a wind farm with power generation is 400 MW, bus 7 is added by a solar site with power generation is $1000 \mathrm{MW}$, and for bus 21 , both a wind farm and a solar site are added with a combined power generation is $300 \mathrm{MW}$. More specifically, the wind power generation is $200 \mathrm{MW}$ and the solar power generation is $100 \mathrm{MW}$.

The total power generation is $4980 \mathrm{MW}$. Therefore, the proportion of IPSs, including the wind and solar power sources with the total power generation is:

$$
\frac{1700}{4980}=34.1 \% \text {. }
$$

\subsection{COPF Simulation Results under Real-Time Dispatch}

Both TOPE and the proposed COPF models are applied under real-time dispatch. Real-time dispatch timescale is chosen to be 1 hour in this simulation. We recorded respectively: the actual power loss, power violation amount and number of buses, and voltage violation amount and number of buses. Simulation results are as follows in Table 1.

\subsection{Discussion}

The results above indicate that TOPF leads to greater power losses and more over-limits when the load trend is descending. TOPF's performance in comparison with COPF varies with the different load trends, with the best one when the load first ascends and then descends, where the over-limits are least. The proposed COPF, on the contrary, generates less over-limits, and performed consistently regardless of the load trend. The COPF alleviates these problems by evaluating the objective function at a more representative point, and taking into consideration the two extreme load points. The COPF is more desirable for its effectiveness.

Table 1. Simulation results of COPF and TOPF models in modified IEEE-30 bus system under real-time dispatch for three load patterns.

\begin{tabular}{cccc}
\hline Load Trend & Parameters & TOPF & COPF \\
\hline \multirow{2}{*}{ Ascend } & Power Violated Amount (MW) & $?$ & $?$ \\
& Voltage Violated Number & 8 & 0 \\
& Voltage Violated Amount (pu) & 0.105 & 0 \\
& Power Loss (MW) & 159.08 & 181.19 \\
Power Violated Amount (MW) & $?$ & $?$ \\
Descend & Voltage Violated Number & 10 & 0 \\
& Voltage Violated Amount (pu) & 0.107 & 0 \\
& Power Loss (MW) & 124.55 & 86.48 \\
& Power Violated Amount (MW) & $?$ & $?$ \\
Ascend then Descend & Voltage Violated Number & 1 & 0 \\
& Voltage Violated Amount (pu) & 0.016 & 115.49 \\
\hline
\end{tabular}




\section{Conclusion}

This paper focuses on optimal dispatch plan that minimizes the total power loss for the power system with high penetration of IPSs. To address security concerns brought by the uncertainties of the IPSs, this paper proposes COPF, which represents the whole time periods better and produces fewer constraint violations. The simulation case studies of the IEEE 30 bus system have shown that COPF is an effective model to generate the optimal dispatch plan for power systems with high penetration of IPSs. While the objective function is minimized, the system security is well satisfied.

\section{Conflicts of Interest}

The author declares no conflicts of interest regarding the publication of this paper.

\section{References}

[1] Nassar, I.A., Hossam, K. and Abdella, M.M. (2019) Economic and Environmental Benefits of Increasing the Renewable Energy Sources in the Power System. Energy Reports, 5, 1082-1088. https://doi.org/10.1016/j.egyr.2019.08.006

[2] Wiser, R.H. and Bolinger, M. (2017) 2016 Wind Technologies Market Report. https://doi.org/10.2172/1375677

[3] Okumoto, Y., Yorino, N., Sasaki, Y., Zoka, Y., Akiyoshi, T. and Isoya, T. (2014) A Study on the Power System Security under Future Uncertainties. IEEJ Transactions on Power and Energy, 134, 949-956. https://doi.org/10.1541/ieejpes.134.949

[4] Rohrig, K. and Lange, B. (2007) Improvement of the Power System Reliability by Prediction of Wind Power Generation. 2007 IEEE Power Engineering Society General Meeting, Tampa, FL, 24-28 June 2007, 1-8. https://doi.org/10.1109/PES.2007.385456

[5] Wang, Z.J. and Guo, Z.Z. (2018) On Critical Timescale of Real-Time Power Balancing in Power Systems with Intermittent Power Sources. Electric Power Systems Research, 155, 246-253. https://doi.org/10.1016/j.epsr.2017.10.022

[6] Wang, Z.J., Guo, G. and Anderson, C.L. (2019) Simulation Case Studies on Period Optimal Power Flow. 2019 Winter Simulation Conference (WSC), National Harbor, MD, 8-11 December 2019, 3669-3680. arXiv preprint arXiv:1908.09879 https://doi.org/10.1109/WSC40007.2019.9004786

[7] Zhang, B., Chen, J., Wu, W.C., Zheng, T., Sun, H.B. and Guo, Q.L. (2012) Real-Time Power Dispatch Method under Wind Power Curtailed Conditions for Large Scale Power Grid. IFAC Proceedings Volumes, 8, 236-241. https://doi.org/10.3182/20120902-4-FR-2032.00043

[8] Bao, Y.-Q., Li, Y., Wang, B.B., Hu, M.Q. and Zhou, Y.M. (2017) Day-Ahead Scheduling Considering Demand Response as a Frequency Control Resource. Energies, 10, 82. https://doi.org/10.3390/en10010082

[9] Wang, Z., Guo, Z., Wang, G. and Wu, Z. (2017) On the Critical Timescale of Real-Time Dispatch Considering Power Balancing under Power Systems with High Proportional Intermittent Power Sources. Proceedings of the Chinese Society of Electrical Engineering, 37, 39-46.

[10] Wang, J.D., Pang, X.K., Gao, S., Zhao, Y. and Cui, S.J. (2019) Assessment of Auto- 
matic Generation Control Performance of Power Generation Units Based on Amplitude Changes. International Journal of Electrical Power \& Energy Systems, 108, 19-30. https://doi.org/10.1016/j.ijepes.2018.12.046

[11] Carpentier, J. (1962) Contribution á l'étude du dispatching économique. Bulletin de la Société Française des Électriciens, 3, 431-447.

[12] Wang, Z.J. and Guo, Z.Z. (2017) Toward a Characteristic Optimal Power Flow Model for Temporal Constraints. 2017 IEEE Transportation Electrification Conference and Expo, Asia-Pacific (ITEC Asia-Pacific), Harbin, 7-10 August 2017, 1-6. https://doi.org/10.1109/ITEC-AP.2017.8081024 